\title{
ON $\tilde{\mho}$-ABNORMAL MAXIMAL SUBGROUPS OF A FINITE SOLVABLE GROUP
}

\author{
PAUL VENZKE
}

\begin{abstract}
Let $\Delta(G)$ be the intersection of the nonnormal maximal subgroup of a finite group. W. Gaschütz has shown that $\Delta(G)$ is nilpotent and that $\Delta(G) / \Phi(G)$ is the center of $G / \Phi(G)$. This note, by considering the intersection of the $\tilde{y}$-abnormal maximal subgroups, generalizes these results for a saturated formation $\mathfrak{F}$.
\end{abstract}

In [2] Gaschütz shows that the intersection of the nonnormal maximal subgroups of the group $G\lrcorner,(G)$, is nilpotent and $\Delta(G) / \Phi(G)=Z(G / \Phi(G))$. In the theory of saturated formations the $\tilde{y}$-abnormal maximal subgroups, introduced by Carter and Hawkes [1], play a role similar to that classically played by the nonnormal maximal subgroups. By considering the intersection of the $\tilde{y}$-abnormal maximal subgroups of a finite group $G$, this note provides a generalization of these results of Gaschütz.

All groups considered in this note are finite and solvable. The notation and terminology is standard. Let $G$ be a finite solvable group and $\tilde{y}=$ $\{\mathfrak{F}(p)\}$ an integrated (i.e. $\widetilde{v} \supseteq \widetilde{F}(p)$ ) local formation. $\Phi(G)$ denotes the Frattini subgroup of $G$ and $G_{\tilde{\pi}}$ denotes the $\tilde{s}$-residual of $G$ (i.e. the smallest normal subgroup of $G$ such that $G / G_{\tilde{\pi}} \in \tilde{\jmath}$ ).

In [1] Carter and Hawkes call a $p$-chief factor $H / K$ of $G$ is-central if $G / C_{G}(H / K) \in \tilde{i}(p)$; otherwise $H / K$ is called $\tilde{F}$-eccentric. They call a maximal subgroup $M$ of $G \tilde{y}$-normal if $M / \operatorname{Core}(M) \in \tilde{y}(p)$ where $p$ is the prime dividing [G:M]; otherwise $M$ is called $\tilde{y}$-abnormal. It is shown [1, Lemma 2.3] that a maximal subgroup $M$ of $G$ is $\tilde{v}$-normal if and only if it complements an $\widetilde{N}$-central chief factor. We note that although in [1] it is assumed all $\widetilde{\mho}(p)$ are nonempty, this condition is not necessary for the above definitions and result.

In [3] Huppert defined the $\tilde{\mho}$-hypercenter of $G, Z_{\tilde{x}}(G)$, to be the largest normal subgroup of $G$ such that all chief factors of $G$ below $Z_{\bar{x}}(G)$ are $\tilde{F}$-central. He shows [3, Satz 1.5] that $Z_{\tilde{y}}(G) \in \tilde{i s}$ whenever the $\tilde{\mho}(p)$ are normal subgroup closed.

LEMMA 1. Let $M$ be a maximal subgroup of $G$. If $M$ is $\mathfrak{y}$-abnormal, then $M \geqq Z_{\tilde{x}}(G)$. If $M$ is $\tilde{i s}$-normal, then $M \geqq G_{\tilde{r}}$.

Received by the editors September 16, 1971.

AMS 1970 subject classifications. Primary 20D10, 20D25; Secondary 20D15, 20D20. Key words and phrases. Solvable group, formation, $\widetilde{y}$-abnormal, $\tilde{i}$-hypercenter.

c. American Mathematical Society 1972 
Proof. Let $M$ be $\widetilde{\mho}$-abnormal and suppose $Z_{\tilde{\mho}}(G) M=G$. Let $R=$ Core $(M)$ and $S=R \cap Z_{\tilde{y}}(G)$. Let $T \leqq Z_{\tilde{y}}(G)$, such that $T / S$ is a chief factor of $G . T / S$ is $\widetilde{\mho}$-central and complemented by $M$. Hence $M$ is $\tilde{\mho}$-normal. $M$ cannot be both $\tilde{\mho}$-normal and $\widetilde{F}$-abnormal, therefore $M \geqq Z_{\mathbb{F}}(G)$.

Let $M$ be $\tilde{\Im}$-normal and suppose $G_{\mathfrak{F}} M=G$. Let $R=\operatorname{Core}(M) . G / R=$ $(M / R)(S / R)$ where $S / R$ is a unique self-centralizing minimal normal subgroup of $G / R$. If $[G: M]=p^{a}$, then $S / R$ is an $\tilde{F}$-central $p$-chief factor of $G$, and $G / S \in \mathfrak{F}(p)$. Since $\mathfrak{\mho} \supseteq \mathfrak{F}(p), G / S \in \mathfrak{F}$ and $S \geqq G_{\mathfrak{\mho}}$. By the Dedekind property $S=G_{\mathfrak{y}}(M \cap S)=G_{\mathfrak{\gamma}} R$. Thus $S / R$ is $G$-isomorphic to $G_{\mathfrak{\S}} / R \cap G_{\mathfrak{\mho}}$ and $G_{\mathfrak{y}} / R \cap G_{\mathfrak{F}}$ is $\tilde{F}$-central. This is impossible; therefore $M \geqq G_{\mathfrak{F}}$.

We denote the intersection of all $\tilde{\mho}$-abnormal maximal subgroups of $G$ by $\Delta_{\tilde{\Upsilon}}(G)$.

THEOREM 1. The following statements are valid.

(a) $\Delta_{\mathfrak{F}}(G) \cap G_{\mathfrak{\mho}} \leqq \Phi(G)$.

(b) $\Delta_{\mathfrak{\gamma}}(G) \geqq Z_{\widetilde{\gamma}}(G)$.

(c) $\Delta_{\mathfrak{i}}(G) / \Phi(G)=Z_{\mathfrak{F}}(G / \Phi(G))$.

Proof. Statements (a) and (b) are an immediate consequence of Lemma 1 , thus we need only show the validity of statement (c).

As $\Delta_{\tilde{f}}(G / \Phi(G))=\Delta_{\tilde{f}}(G) / \Phi(G)$, it follows from statement (b) that $\Delta_{\mathfrak{F}}(G) / \Phi(G) \geqq Z_{\mathfrak{F}}(G / \Phi(G)) . \quad(G / \Phi(G))_{\mathfrak{Y}}=G_{\mathfrak{Y}} \Phi(G) / \Phi(G)$, thus statement (a) implies that $\Delta_{\mathfrak{F}}(G) / \Phi(G) \cap(G / \Phi(G))_{\mathfrak{r}}=\{1\}$. If $H / K$ is a chief factor of $G / \Phi(G)$ which lies below $\Delta_{\mathfrak{F}}(G) / \Phi(G)$, then $H(G / \Phi(G))_{\mathfrak{F}} / K(G / \Phi(G))_{\mathfrak{F}}$ is $G$-isomorphic to $H / K$. Since $H(G / \Phi(G))_{\mathfrak{F}} / K(G / \Phi(G))_{\mathfrak{F}}$ lies above $(G / \Phi(G))_{\mathbb{F}}$, it is $\widetilde{F}$-central and thus $H / K$ is $\widetilde{\mho}$-central. Hence all chief factors of $G / \Phi(G)$ lying below $\Delta_{\tilde{\gamma}}(G) / \Phi(G)$ are $\tilde{F}$-central so that $\Delta_{\mathfrak{F}}(G) / \Phi(G) \leqq Z_{\mathfrak{F}}(G / \Phi(G))$. Therefore $\Delta_{\mathfrak{x}}(G) / \Phi(G)=Z_{\mathfrak{F}}(G / \Phi(G))$.

From statements (a) and (b) above, we see

Corollary. $Z_{\tilde{y}}(G) \cap G_{\tilde{y}} \leqq \Phi(G)$.

We now investigate more closely the structure of $\Delta_{\widetilde{r}}(G)$. For this we let $\pi$ be the set of primes for which the $\tilde{\mho}(p)$ are nonempty. It is useful to present two elementary lemmas. The first of these is a consequence of the conjugacy of $\widetilde{\mho}$-projectors; its proof is omitted.

LEMMA 2. Let $H$ be a normal subgroup of $G$ and $E$ an $\mathfrak{5}$-projector of $H$, then $G=H N$ where $N=N_{G}(E)$.

LEMMA 3. Let $H$ be a normal $\pi$-subgroup of $G$ with $K \unlhd H$ such that $K \leqq \Phi(G) \cap H$. If $H / K \in \mathfrak{F}$, then $H \in \mathfrak{F}$.

Proof. Let $E$ be an $\tilde{\mho}$-projector of $H$. $H=E K$ and, by Lemma 2, $G=H N_{G}(E)=K N_{G}(E)=\Phi(G) N_{G}(E)=N_{G}(E)$. Hence $E \unlhd H$. Since $H$ is a $\pi$-group and $\mathfrak{F}(p) \neq \varnothing$ for $p \in \pi, N_{H}(E)=E$. Therefore $E=H$ and $H \in \mathfrak{F}$. 
THEOREM 2. $\Delta_{\tilde{y}}(G)=P \partial Q$ where:

1. $P$ is a $\pi$-group with $P \geqq Z_{\tilde{\mho}}(G)$ and $P / P \cap \Phi(G) \cong Z_{\tilde{\mho}}(G / \Phi(G))$; and

2. $Q$ is a $\pi^{\prime}$-group with $Q \leqq \Phi(G)$.

Furthermore if the $\mathfrak{F}(p)$ are normal subgroup closed, then $P \in \tilde{F}$.

Proof. Since the $\mathfrak{F}$-central chief factors are necessarily $\pi$-groups, $Z_{\tilde{F}}(G / \Phi(G))$ is a $\pi$-group. By Theorem $1, \Delta_{\mathfrak{F}}(G) / \Phi(G)$ is then a $\pi$-group. Hence from the nilpotence of $\Phi(G)$ we conclude that $\Delta_{\tilde{\gamma}}(G)=P Q$ where $Q$ is a normal Hall $\pi^{\prime}$-subgroup of $\Delta_{\bar{r}}(G)$ with $Q \leqq \Phi(G)$ and $P$ is a Hall $\pi$ subgroup of $\Delta_{\mathfrak{r}}(G)$. By the Frattini argument $G=N_{G}(P) \Delta_{\mathfrak{F}}(G)=$ $N_{G}(P) Q=N_{G}(P)$ so that $P$ is a normal subgroup of $G$. Thus $\Delta_{\mathfrak{f}}(G)=P \oplus Q$.

Since $Z_{\tilde{\gamma}}(G)$ is a $\pi$-group and $\Delta_{\tilde{\gamma}}(G) \geqq Z_{\tilde{\gamma}}(G), P \geqq Z_{\tilde{\gamma}}(G)$. $Q \leqq \Phi(G)$ implies that $\Delta_{\tilde{\gamma}}(G) / \Phi(G)=P / P \cap \Phi(G)$, thus by Theorem $1, P / P \cap \Phi(G)=$ $Z_{\tilde{s}}(G / \Phi(G))$.

If the $\widetilde{F}(p)$ are normal subgroup closed, then by Huppert's result $Z_{\mathfrak{F}}(G / \Phi(G)) \in \tilde{\mho}$. Hence $P / P \cap \Phi(G) \in \tilde{\mho}$ and by Lemma 3 we conclude that $P \in \mathfrak{F}$.

COROLLARY. If for all $p$, the $\tilde{\psi}(p)$ are nonempty and normal subgroup closed, then $\Delta_{\mathfrak{F}}(G) \in \tilde{F}$.

The author would like to thank Professor Ti Yen who made an important contribution to the formulation and proof of Theorem 2.

\section{REFERENCES}

1. Roger Carter and Trevor Hawkes, The $\tilde{s}$-normalizers of a finite soluble group, J. Algebra 5 (1967), 175-202. MR 34 \#5914.

2. Wolfgang Gaschütz, Über die $\Phi$-Untergruppe endlicher Gruppen, Math. Z. 58 (1953), 160-170. MR 15, 285.

3. Bertram Huppert, Zur Theorie der Formationen, Arch. Math. (Basel) 19 (1968), 561-574. MR 39 \#5697.

Department of Mathematics, Minot State College, Minot, North Dakota 58701 